\title{
Direct Tumor Embolization of Sinonasal Unclassified Spindle Cell Sarcoma with Onyx
}

\author{
Ankit Kansal ${ }^{1}$ Prateek Srinet ${ }^{1}$ Richard Peter Manes ${ }^{1}$ \\ ${ }^{1}$ Section of Otolaryngology, Department of Surgery, Yale University \\ School of Medicine, New Haven, Connecticut, United States \\ Address for correspondence Ankit Kansal, MA, Yale School of \\ Medicine, Yale Physicians Building 800 Howard Avenue, Street 4th \\ Floor, New Haven, CT 06519, United States \\ J Neurol Surg Rep 2016;77:e139-e143. \\ (e-mail: ankit.kansal@yale.edu).
}

\begin{abstract}
Keywords

- sinonasal sarcoma

- Onyx

- intraoperative embolization

Objectives To evaluate the use of a new tumor embolization agent, Onyx (Covidien, Dublin, Ireland), for the use of intraoperative embolization of a sinonasal unclassified spindle cell sarcoma.

Methods A 45-year-old female patient presented to the rhinology clinic with a nasal mass. A biopsy revealed a highly vascular mass consistent with a sinonasal unclassified spindle cell sarcoma. Secondary to its extensive vascularity, the patient underwent preoperative transarterial embolization (TAE) before definitive resection. Due to complex vascular anatomy including feeding vessels emanating from intracranial circulation, incomplete embolization was achieved. Subsequently, intraoperative embolization with Onyx at the time of resection was performed.

Results Intraoperative Onyx use resulted in almost complete devascularization of the tumor with decreased risk of intracranial embolization.

Conclusions Intraoperative embolization with Onyx after an incomplete TAE can be a safe and effective method of achieving near-total embolization of sinonasal tumors.
\end{abstract}

\section{Introduction}

Highly vascularized intranasal tumors represent a unique clinical entity, as their surgical management requires adequate visualization in the face of possible significant bleeding. For these tumors, preoperative transarterial embolization (TAE) is a well-established technique for decreasing blood loss. ${ }^{1}$ While this technique certainly reduces intraoperative bleeding, it is often incomplete due to the extensive vascular structure of these tumors and the surrounding vessels. As a result, many surgeons now inject substances such as $n$-butyl cyanoacrylate (NBCA), polyvinyl alcohol (PVA), or other substances intraoperatively to further decrease blood loss. ${ }^{2}$ This technique has most commonly been used for juvenile nasopharyngeal angiofibromas (JNAs). ${ }^{3,4}$ While this technique has shown greater efficacy than TAE alone, there are some risks involved. Most notably, there is a chance that the embolization will not be limited to the tumor, but will cause intracranial embolization as well. ${ }^{5}$ In this case report, we discuss using a novel substance, Onyx18 (ethylene vinyl alcohol), for direct tumor embolization of a sinonasal sarcoma. These tumors have several similarities to JNAs, most notably in their high degree of vascularity. This technique has not been previously described for this type of tumor, though it previously has been utilized for JNAs. ${ }^{6,7}$

Spindle cell sarcoma is a highly vascular heterogeneous tumor that can arise from many sites. When it affects the sinonasal region, it seems to show a predilection for middleaged women and arises most frequently in the superior sinonasal tract. ${ }^{8}$ Lewis et al published an extensive examination of this tumor type in 28 patients. Histologically, a few key characteristics are seen: (1) highly cellular proliferation of uniform, bland spindle cells with the scant matrix with infiltrative growth; (2) prominent hemangiopericytomalike vascular pattern; and (3) concurrent proliferation of surface-type glandular epithelium.
License terms

Stuttgart · New York
DOI http://dx.doi.org/ 10.1055/s-0036-1586210. ISSN 2193-6358.
October 30, 2015 accepted after revision June 19, 2016 


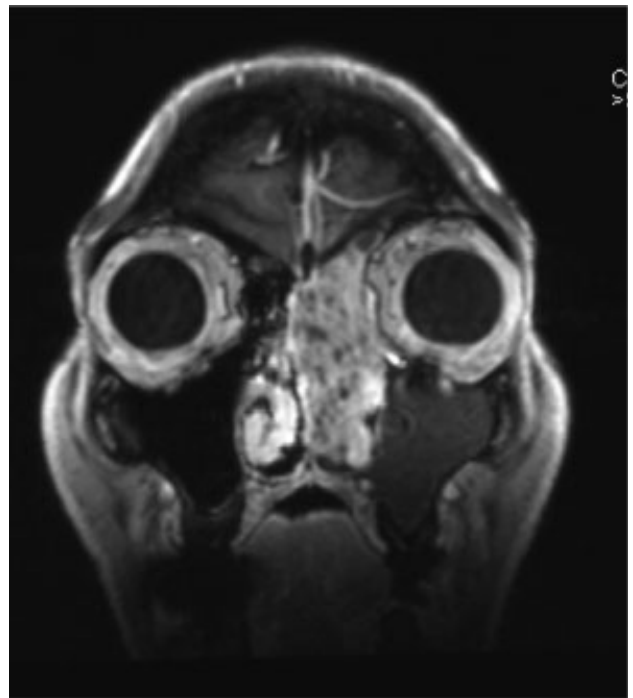

Fig. 1 MRI coronal T1 postcontrast showing sinonasal sarcoma. MRI, magnetic resonance imaging.

\section{Case Presentation}

A 45-year-old female patient presented to the rhinology clinic with a long-standing history of left-sided nasal congestion. In the clinic, rigid nasal endoscopy showed a large nasal mass at the anterior portion of the middle turbinate extending posteriorly in the left nasal cavity. On magnetic resonance imaging (MRI), a large left nasal mass with extension into the ethmoid sinus and abutting the left orbit with multiple flow voids was observed (-Fig. 1). Computed tomography (CT) scan of the paranasal sinuses revealed opacification in the left nasal cavity with the erosion of the nasal septum as well as the cribriform plate (-Fig. 2). There was also some thinning of the left lamina papyracea. When the mass was

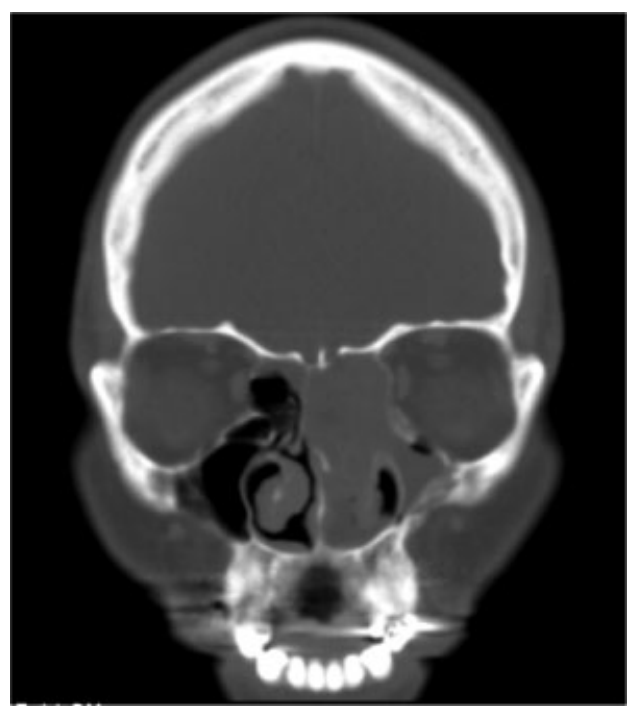

Fig. 2 CT sinus coronal section showing sinonasal sarcoma. CT, computed tomography.

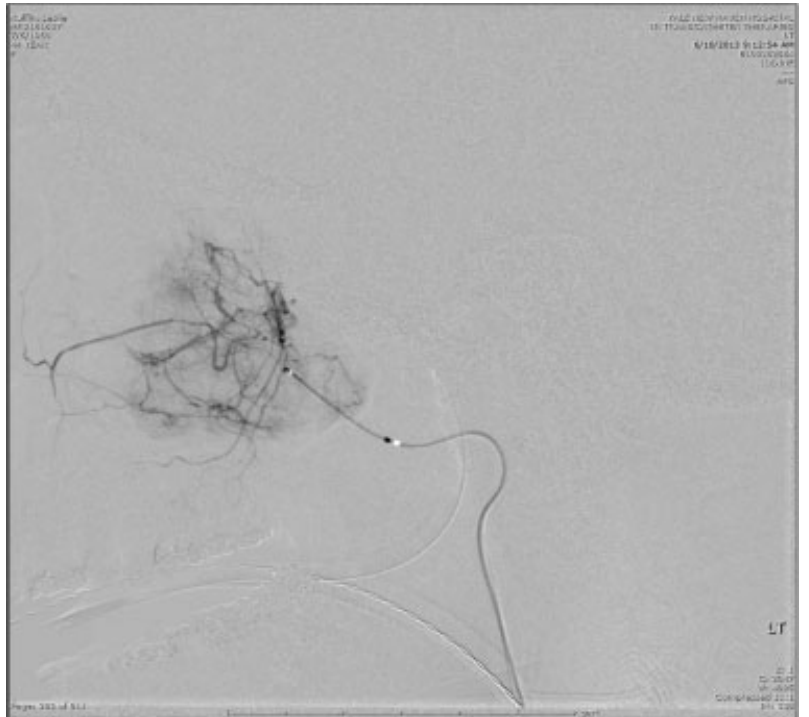

Fig. 3 Pretransarterial embolization showing blood supply from ophthalmic collaterals.

biopsied in the operating room, extensive blood loss was observed. Initial pathology was thought to be a hemangiopericytoma. In planning for endoscopic excision, the patient underwent bilateral internal maxillary artery TAE. Extensive remaining blood supply on the superior aspect of the tumor via ophthalmic artery collaterals was noted on angiography (-Figs. 3 and 4).

\section{Embolization Technique}

Due to the high level of vascularity after transarterial embolization, it was decided to attempt an intraoperative embolization. Using aseptic precautions and under fluoroscopic guidance, a right femoral arterial puncture was performed using a micropuncture technique utilizing a $5 \mathrm{~F}$ sheath (-Figs. 5 and $\mathbf{6}$ ). A 5F

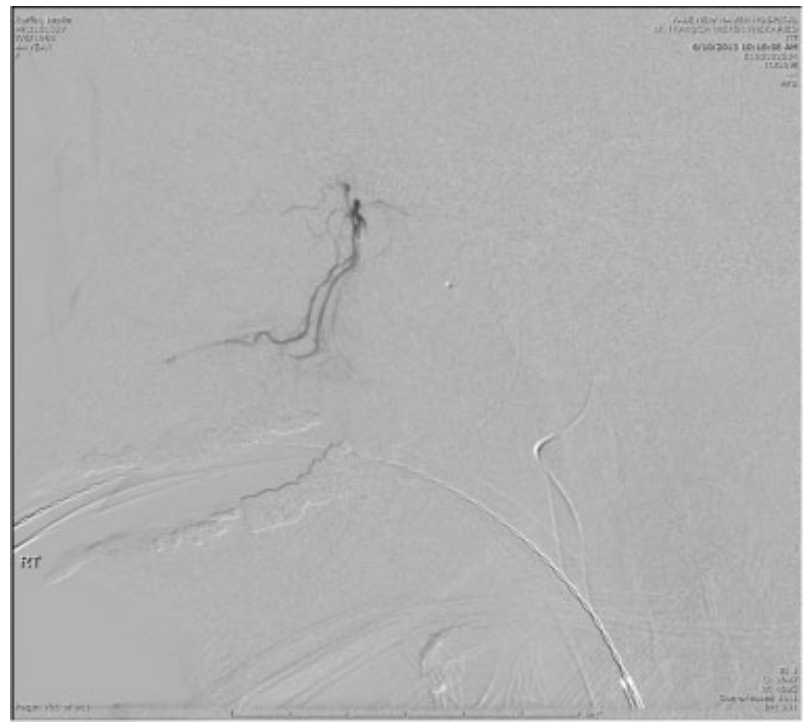

Fig. 4 Posttransarterial embolization showing remaining blood supply. 


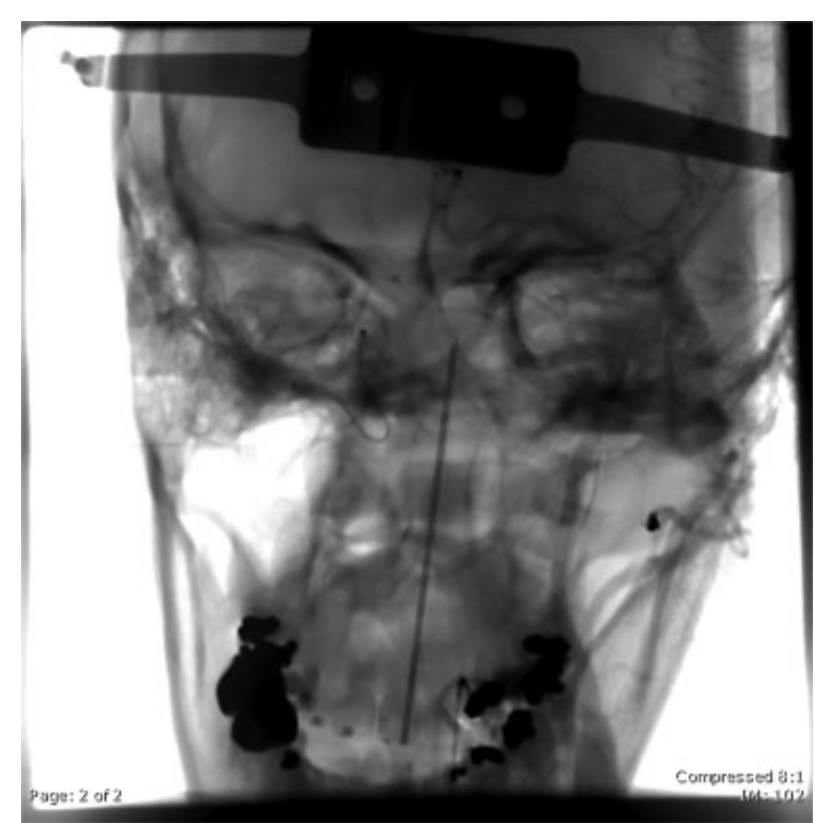

Fig. 5 Coronal view showing intra-arterial embolization.

glide catheter was advanced over a guidewire under fluoroscopic visualization over the aortic arch. The left common carotid artery was catheterized. At this point, oblique and lateral images of the cervical carotid arteries were obtained, and a roadmap was created. Once good visualization was achieved through endoscopy, the tumor was directly punctured with an 18 gauge spinal needle in three different locations. The Onyx liquid embolic system consists of a $1.5 \mathrm{~mL}$ vial of Onyx and a $1.5 \mathrm{~mL}$ vial of dimethylsulfoxide (DMSO), all of which was used. Under fluoroscopic visualization, Onyx-18, an embolic system consisting of ethylene vinyl-alcohol copolymer in DMSO embolization was performed. The predominant filling was observed from the

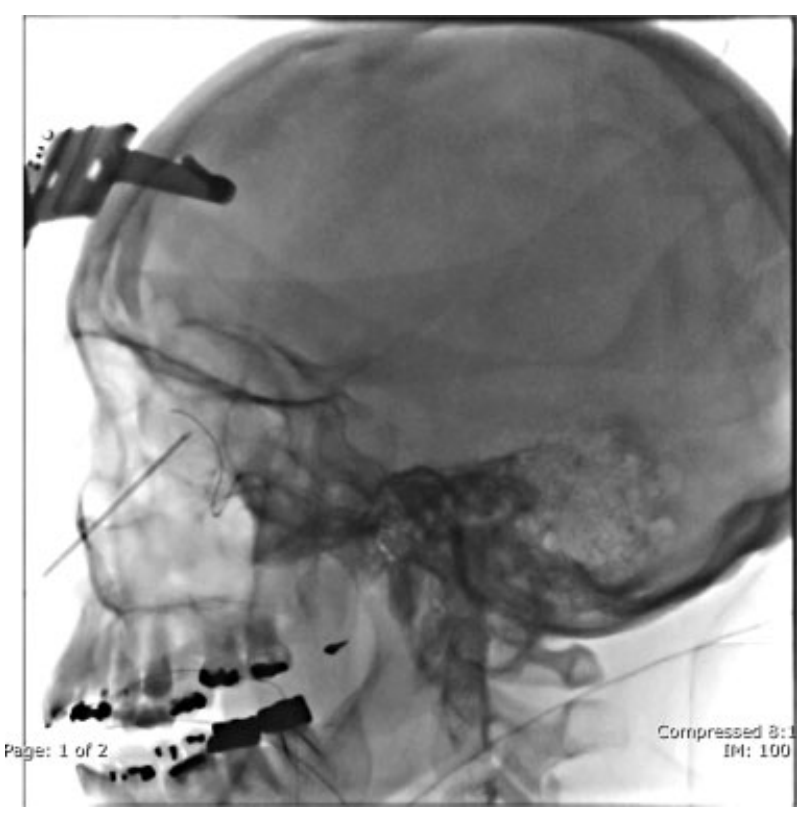

Fig. 6 Sagittal view showing intra-arterial embolization. left internal maxillary artery. In total, 500 microns of Onyx were used. Angiography was utilized to adequately identify the extent of vascularization of the tumor, as well as the feeding vessels, given the significant bleeding encountered on the original biopsy. The fluoroscopy allowed direct observation of the path of the Onyx and stop injection if necessary, which ensured that there was no embolization of any errant vessels, including crucial vessels to the eyes or the brain. The embolization procedure took a total of approximately 15 minutes, including devascularization of the desired vessels. The groin sheath was removed after the final images were obtained. Hemostasis was obtained with a $6 \mathrm{~F}$ Perclose device (Abbott Laboratories, Abbott Park, Illinois).

\section{Results}

As observed on endoscopy, good tumor penetration was achieved via direct tumor puncture without any embolic spill from the mass. Subsequently, the tumor was removed the next day endoscopically with blood loss of $300 \mathrm{~mL}$, despite the dual blood supply from both carotid systems. The sarcoma was then irradiated to $60 \mathrm{~Gy}$ in $2 \mathrm{~Gy}$ fractions. Final pathology classified the mass as an intermediate grade unclassified spindle cell sarcoma, and follow-up MRI after 2 weeks showed no residual disease ( - Fig. 7), and MRI at 2, 8, and 24 months showed no tumor recurrence.

\section{Discussion}

Preoperative embolization is a technique that has been used for many years to reduce intraoperative blood loss in vascular sinonasal tumors. Conventionally, this has been accomplished by transarterial embolization of distal internal maxillary feeders with agents such as NBCA, PVA, or a variety of

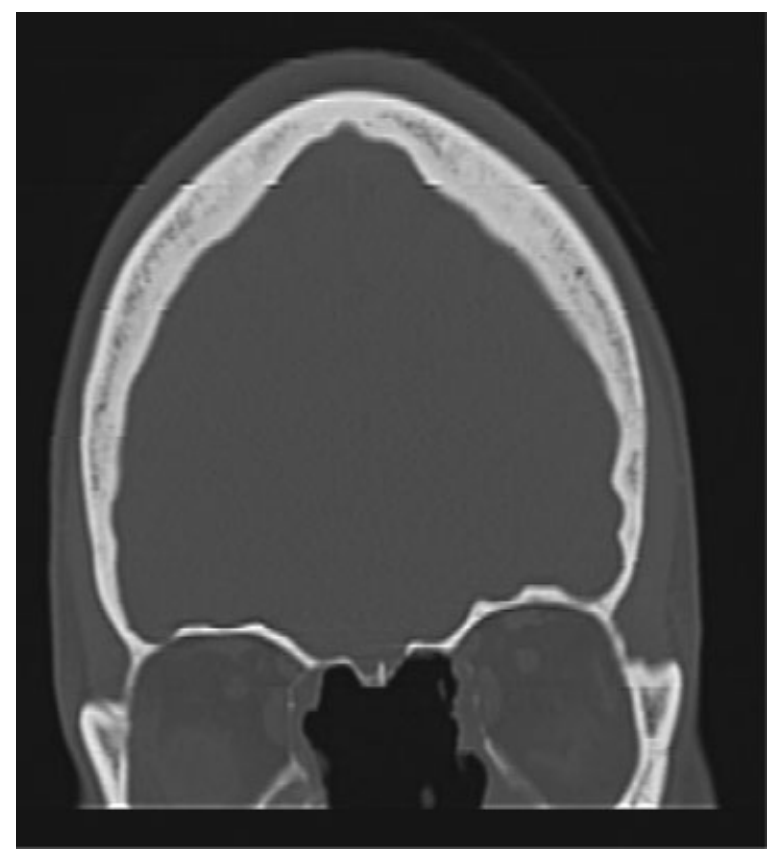

Fig. 7 CT sinus coronal section 1 week postoperatively. CT, computed tomography. 
others. This technique was shown to be effective at reducing blood loss and the rate of transfusion. Li et al showed that by using TAE in JNA's, intraoperative blood loss was reduced from an average of $1,136 \mathrm{~mL}$ in the nonembolized patients to $677 \mathrm{~mL}$ in the embolized patients. In addition, transfusion amounts were reduced from 836 to $400 \mathrm{~mL}{ }^{1}$ However, preoperative TAE does not always achieve complete devascularization, and so direct intratumoral embolization has been used to overcome the limitations of TAE since the early 1990s. This was first shown by Tranbahuy et al, using a small group of seven patients. ${ }^{2}$ In that study, seven patients with JNAs supplied by branches of the internal carotid artery underwent direct intratumoral embolization. Direct tumor puncture caused significantly increased devascularization and necrosis of the tumor, and provided useful perioperative visualization. Patients had no neurological complications from the technique. ${ }^{2}$ Chaloupka et al followed up with a larger study of 26 patients, finding that using direct tumor embolization in conjunction with TAE or alone allowed for successful devascularization in 24 of the 26 cases. ${ }^{4}$ Successful devascularization was categorized as total or near-total devascularization in 20 of the 24 cases, and subtotal devascularization in 4 cases. In those 4 cases, no "poor" or "moderate" grades of devascularization were noted. These grades were determined by the degree of residual neovascularity and parenchymal staining observed on control angiography at the completion of the therapy. The authors then categorized the extent of tumor devascularization by the following grading system: poor (0-30\%); 2 , moderate (30-70\%); 3, subtotal (70-95\%); 4, near-total (95-99\%); and 5, total (100\%). In the two unsuccessful cases, poor visualization of the vascularity of the tumor was noted, suggesting that the TAE had been enough, and no further embolization was necessary.

In the years since that study, it has been shown that this technique has been reliable and can be effective in achieving near-total devascularization compared with TAE. TAE can often remain incomplete due to extensive vascularity and arteriovenous shunting of the lesion. Elhammady et al performed a comparison of TAE versus direct tumor embolization in 10 patients with JNAs, using Onyx in all of them. ${ }^{9}$ The authors measured the percentage of tumor devascularization by tracing the preembolization and postembolization tumor blush using Image software. They also measured the estimated blood loss for both groups of patients. They found that TAE resulted in $77 \%$ devascularization, on average, while direct tumor embolization resulted in 93\% devascularization. They also found that the TAE group had a mean estimated blood loss (EBL) of $862 \mathrm{~mL}$, while the direct tumor embolization group had a mean EBL of $412 \mathrm{~mL}$, though, with the small sample size, this did not reach statistical significance. Nonetheless, it appears that direct embolization can be substantially more effective in achieving devascularization compared with TAE. However, while this technique is effective, there is a significant risk of inadvertent intracranial embolization. In the largest study of direct intratumoral NBCA embolization, Casasco et al evaluated 65 patients with various head and neck tumors. ${ }^{5}$ This study identified two complications: in one patient, there was reflux into the right middle cerebral artery with subsequent infarct and death and in the other, there was reflux into a collateral vessel of the ophthalmic artery with acute loss of vision in that eye. These cases demonstrate both the significant level of risk and the high degree of morbidity and mortality that can occur with traditional direct tumor embolization.

In this patient, we found that Onyx has advantages over other particulate or liquid embolic. Onyx has been used for several years for various tumors, aneurysms, and arteriovenous malformations (AVM). The material is an ethylene vinyl alcohol copolymer dissolved in the organic solvent DMSO and mixed with suspended micronized tantalum powder, which provides contrast for visualization under fluoroscopy. Once the solution comes into contact with an ionic solution, the DMSO dissipates and the Onyx solidifies into a spongy, cohesive material. Because of its cohesive properties, it solidifies more slowly than NBCA, which allows injections to be performed over much longer time intervals with improved control. In addition, although the embolization goes more slowly, the overall operative time is reduced significantly, since the bleeding is reduced. Aziz-Sultan et al first reported the benefits of Onyx for JNAs, but to our knowledge, this technique has not been described for intranasal sarcomas. ${ }^{6}$ Intraoperative embolization has shown efficacy in reducing vascularization, and Onyx has shown benefits through better control of embolization. There is also evidence that Onyx might be more effective for preoperative TAE than classic agents such as NBCA. In one study by Gemmete et al, it was found that Onyx achieved a higher degree of devascularization for JNAs when used preoperatively compared with other agents. ${ }^{10}$ In addition, Onyx has been found to be useful in a variety of tumors, such as hemangiopericytomas. ${ }^{11}$

\section{Conclusion}

We present a case of direct tumor embolization of a sinonasal sarcoma utilizing Onyx. Direct tumor embolization provided near-total devascularization of the sinonasal sarcoma, allowing for endoscopic removal with adequate visualization and reasonable blood loss. We found that Onyx was both safe and effective at achieving devascularization in this type of tumor, even when the vasculature is complicated and TAE is insufficient.

\section{References}

1 Li JR, Qian J, Shan XZ, Wang L. Evaluation of the effectiveness of preoperative embolization in surgery for nasopharyngeal angiofibroma. Eur Arch Otorhinolaryngol 1998;255(8):430-432

2 Tranbahuy P, Borsik M, Herman P, Wassef M, Casasco A. Direct intratumoral embolization of juvenile angiofibroma. Am J Otolaryngol 1994;15(6):429-435

3 Gullane PJ, Davidson J, O’Dwyer T, Forte V. Juvenile angiofibroma: a review of the literature and a case series report. Laryngoscope 1992;102(8):928-933

4 Chaloupka JC, Mangla S, Huddle DC, et al. Evolving experience with direct puncture therapeutic embolization for adjunctive and palliative management of head and neck hypervascular neoplasms. Laryngoscope 1999;109(11):1864-1872 
5 Casasco A, Houdart E, Biondi A, et al. Major complications of percutaneous embolization of skull-base tumors. AJNR Am J Neuroradiol 1999;20(1):179-181

6 Aziz-Sultan MA, Moftakhar R, Wolfe SQ, Elhammady MS, Herman $B$, Farhat $H$. Endoscopically assisted intratumoral embolization of juvenile nasopharyngeal angiofibroma using Onyx. J Neurosurg Pediatr 2011;7(6):600-603

7 Lehmann M, Ulrich S, Reineke U, Hamberger U, Dietrich U, Sudhoff $\mathrm{H}$. [Intratumoral Onyx embolisation in the management of juvenile nasopharyngeal angiofibroma]. HNO 2010;58(8):853-857

8 Lewis JT, Oliveira AM, Nascimento AG, et al. Low-grade sinonasal sarcoma with neural and myogenic features: a clinicopathologic analysis of 28 cases. Am J Surg Pathol 2012;36(4):517-525
9 Elhammady MS, Johnson JN, Peterson EC, Aziz-Sultan MA. Preoperative embolization of juvenile nasopharyngeal angiofibromas: transarterial versus direct tumoral puncture. World Neurosurg 2011;76(3-4):328-334, discussion 263-265

10 Gemmete JJ, Patel S, Pandey AS, et al. Preliminary experience with the percutaneous embolization of juvenile angiofibromas using only ethylene-vinyl alcohol copolymer (Onyx) for preoperative devascularization prior to surgical resection. AJNR Am J Neuroradiol 2012;33(9):1669-1675

11 Ledderose GJ, Gellrich D, Holtmannspötter M, Leunig A. Endoscopic resection of sinonasal hemangiopericytoma following preoperative embolisation: A case report and literature review. Case Rep Otolaryngol 2013;2013:796713 